\title{
Multichannel SQUID Magnetometry Using Double Relaxation Oscillation SQUID's
}

\author{
Michiel J. van Duuren, Y. H. Lee, Derk Jan Adelerhof, J. Kawai, H. Kado, Jaap Flokstra, and Horst Rogalla
}

\begin{abstract}
A highly sensitive first-order gradiometer based on double relaxation oscillation SQUID's (DROS's) for multichannel use is presented. The white flux noise level of the bare DROS's is $4.5 \mu \Phi_{0} / \sqrt{\mathbf{H z}}(\varepsilon=275 \mathbf{h})$. With wire-wound first-order gradiometers, having a baseline of $40 \mathrm{~mm}$, the white magnetic field noise equals $4 \mathrm{fT} / \sqrt{\mathrm{Hz}}$. As a result of the high flux-tovoltage transfer of the DROS's of about $1 \mathrm{mV} / \Phi_{0}$, this low noise level could be obtained with simple room-temperature flux locked loop electronics based on direct voltage readout. The relaxation frequency of the present DROS's is approximately $1 \mathrm{GHz}$. No degradation of the DROS characteristics caused by interference between relaxation oscillations in two adjacent channels has been observed, although the gradiometers are spaced by less than $1 \mathrm{~mm}$. This shows that DROS's can be used in multichannel SQUID magnetometers.
\end{abstract}

\section{INTRODUCTION}

$\mathbf{T}$ THE UNRIVALLED magnetic flux sensitivity of low $T_{c}$ dc SQUID's allows measurements that are impossible with other sensors. In biomagnetic measurements for human brain research, for example, magnetic fields below $10^{-13} \mathrm{~T}$ have to be detected. Conventional SQUID magnetometers are based on nonhysteretic dc SQUID's, which have a typical flux noise level of $1-10 \mu \Phi_{0} / \sqrt{\mathrm{Hz}}$, where $\Phi_{0}=2.07 \cdot 10^{-15} \mathrm{~Wb}$ is the flux quantum, and a flux-to-voltage transfer of 10-100 $\mu \mathrm{V} / \Phi_{0}[1],[2]$. This results in an output voltage noise of about $0.1 \mathrm{nV} / \sqrt{\mathrm{Hz}}$, which is much lower than the input voltage noise of $1 \mathrm{nV} / \sqrt{\mathrm{Hz}}$ that can be obtained with low noise dc amplifiers at room temperature. To prevent amplifier-limitation of the sensitivity of a dc-SQUID magnetometer, flux modulation techniques in combination with cryogenic impedance matching circuitry are frequently used. In multichannel applications, these modulation schemes exhibit some severe drawbacks, such as limited bandwidth, crosstalk between adjacent channels, and complicated electronics. Therefore, several groups have developed new SQUTD types, like dc SQUID's with additional positive feedback (APF) [3], [4], two-stage SQUID's in which the signal of the sensing SQUID is amplified by a second SQUID [5], [6], or by a series array of SQUID's [7], single-chip digital SQUTD's [8], relaxation oscillation SQUID's (ROS's) [9]-[13], and double relaxation

Manuscript received September 7, 1995; revised January 23, 1996.

M. J. van Duuren, D. J. Adelerhof, J. Flokstra, and H. Rogalla are with the Department of Applied Physics, Low Temperature Division, University of Twente, 7500 AE Enschede, The Netherlands.

Y. H. Lee is with the Korean Research Institute of Standards and Science, Taedok Science Town, Taejon 305-606 Korea.

J. Kawai and H. Kado are with the Superconducting Sensor Laboratory, Chiba 270-13 Japan.

Publisher Item Identifier S 1051-8223(96)03122-3. oscillation SQUTD's (DROS's) [12]-[14]. All these SQUID's enable direct readout with relatively simple room-temperature electronics. This paper deals with DROS's.

A DROS converts a magnetic flux to a volfage with a flux-to-voltage transfer that is typically $10-100$ times larger than that of conventional de SQUID's. This large transfer enables a simple flux locked loop (FLL) configuration, based on direct voltage readout at room temperature. The principle of operation is based on relaxation oscillations that are induced in hysteretic dc SQUID's. At sufficiently high relaxation frequencies, 1 to $3 \mathrm{GHz}$ typically, the intrinsic noise level of DROS's is the same as that of nonhysteretic dc SQUID's [15], [16]. Recently, it has been shown that the magnetic field sensitivity of a DROS gradiometer is sufficient to detect magnetic brain signals [17]. However, until now, it was not clear whether the relaxation oscillations of one DROS channel interfere with those of an adjacent channel. This would limit the application of DROS's in multichannel magnetometers. In this paper, it is shown that the magnetic field noise level of a first-order DROS gradiometer does not depend on the operation of an adjacent DROS gradiometer channel.

\section{THEORY OF OPERATION}

A DROS consists of two dc SQUID's in series, shunted by a resistor $R_{s h}$ and an inductor $L_{s h}$, as shown schematically in Fig. 1. The dc SQUID's are based on underdamped Josephson tunnel junctions, thus causing hysteretic SQUID I-V curves. The magnetic flux to be measured, $\Phi$, is applied to the upper SQUID, the signal SQUID. An adjustable reference flux, $\Phi_{\text {ref }}$, is applied to the lower SQUID, the reference SQUID. If the DROS is biased with a dc bias current $I_{b}$, relaxation oscillations can occur. During these relaxation oscillations, only the SQUID with the smallest critical current switches to the voltage state. To a good approximation, the relaxation frequency $f_{r o}$ is given by

$$
f_{r o}=\tau_{s h}^{-1} \cdot \ln ^{-1}\left(\frac{I_{b}}{I_{b}-I_{c}(\Phi)}\right)
$$

In (1), $\tau_{s h}=L_{s h} / R_{s h}$ is the time constant of the $L$ $R$ shunt and $I_{c}(\Phi)$ is the smallest of the two SQUTD critical currents. In each relaxation oscillation cycle, the critical current of the signal SQUID, $I_{c, \operatorname{sign}}(\Phi)$, is compared with the constant critical current of the reference SQUID, $I_{c, \text { ref }}\left(\Phi_{\text {ref }}\right)$. If $I_{c, \text { sign }}(\Phi)<I_{c, \text { ref }}\left(\Phi_{\text {ref }}\right)$, the signal SQUID switches to the finite voltage state during the relaxation 


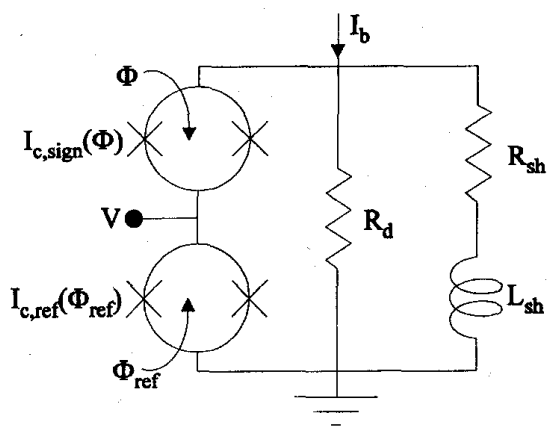

Fig. 1. Schematic overview of a DROS.

oscillations, whereas the reference SQUID stays in the zerovoltage state, so no voltage will be present between the voltage output terminal $V$ and ground. However, if $\Phi$ is changed such that $I_{c, \text { sign }}(\Phi)>I_{c, \text { ref }}\left(\Phi_{\text {ref }}\right)$, the reference SQUID switches during the oscillations, causing a sequence of voltage pulses at the output. The time-averaged value of these pulses is $V_{c}=V_{c}\left(I_{b}, \Phi_{\text {ref }}\right)$. Typically, for $I_{b}$ well above $I_{c, \text { ref }}, V_{c}=(0.5-0.75) \cdot I_{b} \cdot R_{s h}$. To assure that both the situations $I_{c, \text { sign }}>I_{c, \text { ref }}$ and $I_{c, \text { sign }}<I_{c, \text { ref can occur if the }}$ signal flux $\Phi$ changes, $I_{c \text {,ref }}$ has to be adjusted to a value in the centre of the $I_{c, \text { sign }}$ modulation range, simply by varying $\Phi_{\text {ref. }}$. The result is a $V-\Phi$ characteristic with a very high transfer $\partial V / \partial \Phi$ at the transition from $V=0$ to $V=V_{c}$, where the critical currents of both SQUID's are equal.

The relaxation oscillations are persistent if the following operation condition is satisfied [15]:

$$
\beta_{c}^{*} \cdot\left(\frac{I_{b}}{2 I_{0}}\right)^{2}<1 \text { with } \beta_{c}^{*}=\frac{2 \pi \cdot 2 I_{0} \cdot R_{s h}^{2} \cdot C_{s q}}{\Phi_{0}} .
$$

In (2), $I_{0}$ is the junction critical current, $C_{s q}$ the SQUID capacitance, and $\beta_{c}^{*}$ the effective McCumber parameter for a DROS. Combined with $V_{c}=(0.5-0.75) \cdot I_{b} \cdot R_{s h}$, (2) yields the following expression for the maximum voltage modulation depth $\Delta V\left(=V_{c}\right)$ that can be achieved with a DROS:

$$
\begin{aligned}
\Delta V_{\mathrm{DROS}, \max } & =(0.5 \cdots 0.75) \cdot I_{b, \max } \cdot R_{s h} \\
& =(0.5 \cdots 0.75) \cdot \sqrt{\frac{2 I_{0} \cdot \Phi_{0}}{2 \pi \cdot C_{s q}}} .
\end{aligned}
$$

Equation (3) shows that the voltage modulation depth of a DROS does not depend on $R_{s h}$, thus relaxing the design and fabrication constraints.

To prevent $L-C$ resonances in the $L-C-R$ loop formed by $L_{s h}, C_{s q}$, and $R_{s h}$, an additional damping resistor $R_{d}$ can be included as shown in Fig. 1. The $L_{s h}-C_{s q}$ oscillations that can deteriorate the DROS characteristics drastically are adequately damped if the damping parameter $D$ satisfies the following:

$$
D=\frac{L_{s h}}{4 \cdot C_{s q} \cdot R_{d}^{2}}>1
$$

Since, in practical cases, $R_{d} \gg R_{s h}$, addition of $R_{d}$ does not affect the operation criterion given by (2).
For relaxation frequencies well below the plasma frequency of the tunnel junctions (by a factor of about 50 or more), the flux-to-voltage transfer $\partial V / \partial \Phi$ of a sufficiently damped DROS is mainly determined by $V_{c}$ and by the thermal spread on the critical currents of both SQUID's. Numerical simulations yield the following analytical expression for the thermally limited flux-to-voltage transfer [12], [15]:

$$
\left(\frac{\partial V}{\partial \Phi}\right)_{\max }=7 \cdot \frac{I_{0}^{2 / 3}}{\left(1+\beta_{L, \text { sign }}\right)} \cdot \frac{V_{c}}{\Phi_{0}} .
$$

In this equation, $I_{0}$ is the junction critical current in microamperes and $\beta_{L, \text { sign }}=2 I_{0} \cdot L_{s q, \text { sign }} / \Phi_{0}$, with $L_{s q, \text { sign }}$ being the signal SQUID inductance. Numerical simulations showed that for much higher relaxation frequencies, (5) no longer holds true.

At the points of maximum transfer, the switching probability for both SQUID's is $50 \%$. From the voltage noise spectrum caused by this stochastic process and from (5), the following expression for the flux noise spectral density of a DROS can be derived [12], [15]:

$$
\sqrt{S_{\Phi}}=2.3 \cdot \frac{\left(1+\beta_{L, \mathrm{sign}}\right)}{I_{0}^{2 / 3}} \cdot \sqrt{\frac{1}{f_{\text {ro }}}} .
$$

In (6), $\sqrt{S_{\Phi}}$ is expressed in $\mu \Phi_{0} / \sqrt{\mathrm{Hz}}$ if the junction critical current $I_{0}$ is substituted in microamperes and the relaxation frequency $f_{r o}$ in GHz. From (6), it turns out that DROS's can have the same sensitivity as comparable nonhysteretic dc SQUID's if the relaxation frequency is sufficiently high, of the order of $1 \mathrm{GHz}$ typically.

\section{EXPERIMENTAL SETUP}

A three-channel insert for use in a fiber glass liquid helium cryostat (BTI model BMD 5) has been constructed. Most measurements were done in a magnetically shielded room (MSR, Vacuumschmelze, type AK 3B). The three wire-wound firstorder gradiometers are positioned in a triangular configuration, and are spaced less than $1 \mathrm{~mm}$, which is small compared to the pickup coil diameter of $20 \mathrm{~mm}$. The gradiometers touch the bottom of the cryostat; to prevent mechanical tensions during cooling down, they can move in axial direction. About $15 \mathrm{~cm}$ above the pickup section, three DROS's are mounted in separate cylindrical $\mathrm{Nb} / \mathrm{Pb}$ modules with an outer diameter of $10 \mathrm{~mm}$ and a length of $5 \mathrm{~cm}$. The connection between each DROS-module and the room-temperature FLL electronics is made by resistive wires ( $R \approx 25 \Omega$ per wire), twisted in pairs and shielded by a stainless steel tube. Each channel has its own FLL electronics in a separate aluminum box directly on top of the cryostat. The data acquisition system, outside the MSR, is connected to the system with coaxial cables.

\section{A. DROS Layout}

Fig. 2(a) shows a micrograph of the DROS's that were used, whereas Fig. 2(b) gives a schematic diagram. The $\mathrm{Nb} / \mathrm{Al}, \mathrm{AlO}_{x} / \mathrm{Nb}$ Josephson tunnel junctions, other $\mathrm{Nb}$ 


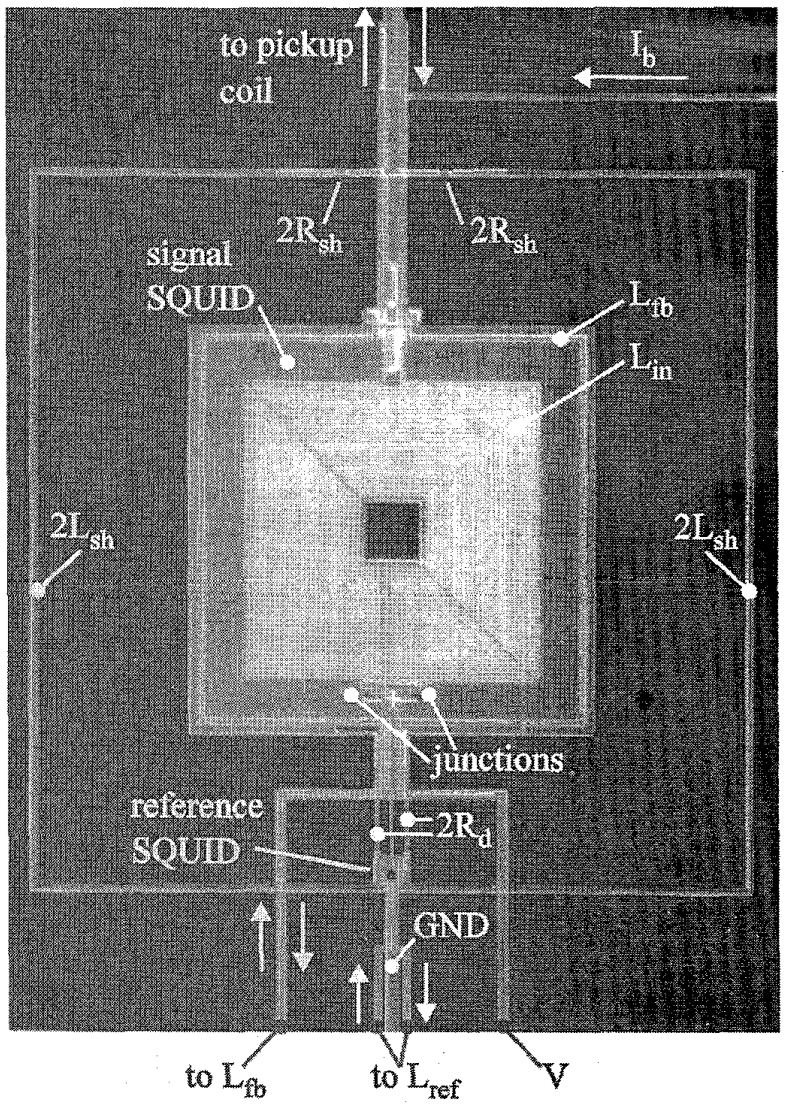

(a)

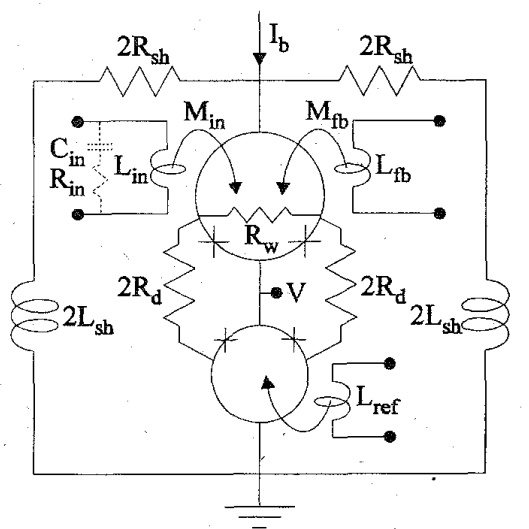

(b)

Fig. 2. (a) Micrograph of an actual DROS. (b) Scheme of the actual DROS layout.

structures and the $\mathrm{SiO}_{2}$ insulating layers are patterned by reactive ion etching (RIE) in $\mathrm{CF}_{4}$. Resistors are structured in Al thin films by wet chemical etching in a $\mathrm{NaOH}$ containing solution.

The washer type signal SQUID is tightly coupled to a 25 turns input coil. The hole width is $100 \mu \mathrm{m}$, resulting in the theoretical values $L_{\text {hole }}=160 \mathrm{pH}$ and $L_{\text {in }}=100 \mathrm{nH}$ [18]. A single-turn feedback coil is included at the outside of the input coil. From the experimental $I_{c, \text { sign }}(\Phi)$ modulation, $\beta_{L, \text { sign }}$ was estimated to be 1.3 , giving $L_{s q \text {, sign }}=240 \mathrm{pH}$. Combined with $L_{\text {hole }}$, this value indicates $80 \mathrm{pH}$ of parasitic inductance, probably caused by the double slit, which has a length of $250 \mu \mathrm{m}$ and a slit width of $2 \mu \mathrm{m}$. The mutual inductance between the signal SQUID and the input coil, $M_{\text {in }}$, was measured to be $4.1 \mathrm{nH}$. With the experimental SQUTD inductance of $L_{s q}=240 \mathrm{pH}$ and the design value $L_{\text {in }}=100 \mathrm{nH}$, this yields a coupling coefficient between SQUID washer and input coil of 0.84 . To prevent $L-C$ resonances in the signal SQUID, the SQUID inductance is shunted with a resistor, $R_{w}$, of $3 \Omega$ [19]. Since $R_{w}$ is smaller than the characteristic SQUID impedance, given by $\frac{1}{2} \sqrt{\left(L_{s q} / \frac{1}{4} C_{s q}\right)}, L-C$ resonances in the signal SQUID are adequately damped. On the other hand, $R_{w}$ is not so small that its Johnson noise significantly increases the intrinsic flux noise of the DROS: $L_{s q} \cdot i_{n}=L_{s q} \cdot \sqrt{4 k T / R_{w}} \approx 1 \mu \Phi_{0} / \sqrt{\mathrm{Hz}}$. The hysteretic $2 \times 2 \mu \mathrm{m}^{2}$ Josephson junctions at the outer side of the signal SQUDD washer have a capacitance of $0.25 \mathrm{pF}$ each, and a critical current $I_{0}$ of $5.5 \mu \mathrm{A}$.

The much smaller reference SQUID has a designed inductance of $32 \mathrm{pH}$. A single turn coil is integrated on top of the reference SQUID to generate the reference flux, $\Phi_{\text {ref }}$. The $2.2 \times 2.2 \mu \mathrm{m}^{2}$ junctions have $I_{0}=6.5 \mu \mathrm{A}$. The small SQUDD inductance gives $\beta_{L \text {,ref }} \approx 0.2$, which enables a wide modulation range for $I_{c, \text { ref }}$, covering the $I_{c, \text { sign }}$ modulation range completely.

The inductive shunt, which has a design value of $L_{s h}=1$ to $1.5 \mathrm{nH}$, is located symmetrically around both SQUID's, to prevent possible negative effects of magnetic coupling between the shunt inductance and the SQUID's. Each branch has its own resistor of $2 \Omega$, so that $R_{s h}=1 \Omega$, implying $\beta_{c}^{*}=0.017$. This small $\beta_{c}^{*}$ ensures proper operation of the DROS, even for relatively high bias currents: according to (2), $I_{b, \max }=2 I_{0} / \sqrt{\beta_{c}^{*}} \approx 85 \mu \mathrm{A}$, yielding a theoretical voltage modulation depth $\Delta V$ of about $50 \mu \mathrm{V}$. According to (1), the relaxation frequency is of the order of $1 / \tau_{s h}=R_{s h} / L_{s h}$, so around $1 \mathrm{GHz}$ for moderate bias currents. According to (6), this value of $f_{\text {ro }}$ yields a theoretical flux noise level of $1.7 \mu \Phi_{0} / \sqrt{\mathrm{Hz}}$.

Resonances between the SQUID capacitance and the shunt inductance are damped by two damping resistors of $30 \Omega$ each, yielding $D=2-3$. The damping resistors are connected as shown in Fig. 2(b) in order to shunt the junction capacitance as directly as possible: the inductance of both SQUID loops is not a part of the damping circuit.

\section{B. Flux Transformers}

The flux transformers consist of first-order $\mathrm{Nb}$ wire-wound gradiometers, connected to the 25 turns input coils of the DROS's. The gradiometers, which are wound around a textile reinforced epoxy core, consist of two sensing turns and two compensation turns, separated by the baseline of $40 \mathrm{~mm}$. The sensing turns have an intermediate distance of $1 \mathrm{~mm}$ whereas the two compensation turns are spaced by $12 \mathrm{~mm}$ in order to 


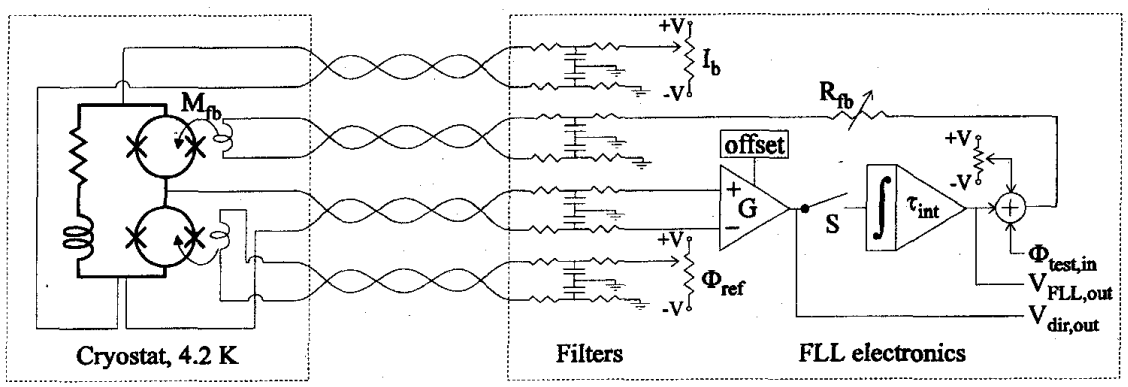

Fig. 3. Simplified DROS flux locked loop with direct voltage read-out at room temperature.

minimize the inductance $L_{p u}$ of the gradiometer. The diameter of the gradiometers is $20 \mathrm{~mm}$, resulting in a calculated self inductance $L_{p u}$ of $0.35 \mu \mathrm{H}$ and a sensing area $A_{p u}$ of $6.3 \mathrm{~cm}^{2}$. Inside the DROS modules, the twisted insulated $\mathrm{Nb}$ wires coming from the gradiometers are connected to $50 \mu \mathrm{m}$ thick $\mathrm{Nb}$ bonding wires by means of small $\mathrm{Nb}$ blocks with screws. The superconducting connection with the input coil is made by ultrasonic bonding of these $50 \mu \mathrm{m}$ thick wires, that have to be pretreated as described in [20] prior to bonding.

Across the input coil pads, an external $R-C$ shunt, consisting of a $150 \Omega$ metal film resistor, $R_{\text {in }}$, in series with a $1-\mathrm{nF}$ capacitor, $C_{\mathrm{in}}$, is connected [17]. When the gradiometers are connected, the $R_{\text {in }}-C_{\text {in }}$ shunt forms a $250-300 \mathrm{MHz}$ low pass filter, preventing external high frequency magnetic noise to interfere with the relaxation oscillations.

\section{FLL Electronics}

A schematic overview of the FLL electronics for one channel is given in Fig. 3. At the top of the insert, all leads are lowpass filtered with respect to ground by means of simple $R-C$ filters. The cutoff frequency of the filters in the bias current and reference flux leads is $2 \mathrm{kHz}$, whereas the filters in the voltage and feedback flux leads have cutoff frequencies of $3 \mathrm{MHz}$, to enable a high bandwidth in FLL. The output voltage of the DROS is fed to a differential instrumentation amplifier based on LT 1028 op-amps. The gain of this amplifier can be adjusted from $G=2 \cdot 10^{2}$ to $G=2 \cdot 10^{5}$. The white input voltage noise level is about $1.8 \mathrm{nV} / \sqrt{\mathrm{Hz}}(5 \mathrm{nV} / \sqrt{\mathrm{Hz}}$ @ $1 \mathrm{~Hz}$ ), whereas the white current noise amounts about $2 \mathrm{pA} / \sqrt{\mathrm{Hz}}(30 \mathrm{pA} / \sqrt{\mathrm{Hz}} @ 1 \mathrm{~Hz})$. To minimize the effect of current noise, especially for low frequencies, the total resistance in the voltage leads, including filtering, has been kept below $80 \Omega$. The output of the amplifier, which has a bandwidth of about $250 \mathrm{kHz}$, is connected to an integrator with an integration time $\tau_{\text {int }}=10^{-2}, 10^{-3}$ or $10^{-4} \mathrm{~s}$. The integrator output provides the feedback flux for the DROS via an adjustable feedback resistor $\left(R_{f b}\right)$. In practice, $R_{f b}$ was adjusted to such a value that $R_{f b} / M_{f b}=1 \mathrm{~V} / \Phi_{0}$. For testing purposes, e.g., $V-\Phi$ characterization in open loop configuration (switch $S$ opened), a test flux, $\Phi_{\text {test }}$, and an offset flux can be added to the feedback flux.
The simple layout of this FLL allows a large small signal bandwidth $f_{c, F L L}$ :

$$
f_{c, \mathrm{FLL}}=\frac{G \cdot\left(\frac{\partial V}{\partial \Phi}\right)_{\mathrm{DROS}} \cdot M_{f b}}{2 \pi \cdot \tau_{\text {int }} \cdot R_{f b}} .
$$

For large signals $\left(>\frac{1}{2} \Phi_{0}\right)$ the maximum slew rate of the FLL is important:

$$
\left(\frac{\partial \Phi}{\partial t}\right)_{\max }=\frac{\frac{1}{2} \Delta V_{\mathrm{DROS}} \cdot G \cdot M_{f b}}{\tau_{\mathrm{int}} \cdot R_{f b}} .
$$

The three channels have one common power supply, outside the shielded room. The powerlines are split inside the MSR, to prevent excessive $50 \mathrm{~Hz}$ noise arising from ground loops. The ground leads of all cables traversing the wall of the MSR are connected to the MSR, as is the insert itself. With this grounding scheme, the system works even when the door of the MSR is open.

\section{EXPERIMENTAL RESUlTS}

The experiments that have been performed had three main issues: characterization of the flux transformers, performance tests of a single DROS gradiometer channel, and investigations of the simultaneous operation of two adjacent DROS gradiometers. Below, the results are discussed in this order.

\section{A. Flux Transformers}

DROS $V-\Phi$ curves have been recorded by feeding a current $I_{f b}$ through the feedback coils of the DROS's. In Fig. 4, $V-\Phi$ characteristics are shown for three different cases: in the first trace (Fig. 4(a)), only the $R_{\mathrm{in}}-C_{\mathrm{in}}$ shunt is connected to the input coil; the second trace (Fig. 4(b)) gives the $V-\Phi$ curve in the case of a superconductively shorted input coil; and the last trace (Fig. 4(c)) was recorded with connected first-order gradiometer. The gradiometers were shielded by a lead can around them, which was necessary to suppress the effect of Johnson noise in the radiation shields of the cryostat: preliminary measurements showed a noise level of about $30 \mathrm{fT} / \sqrt{\mathrm{Hz}}$ when the gradiometers touched the bottom of the cryostat, decreasing significantly when the gradiometers were lifted a few centimeters above the bottom. As can be seen from the different periods of the traces in Fig. 4, the mutual 


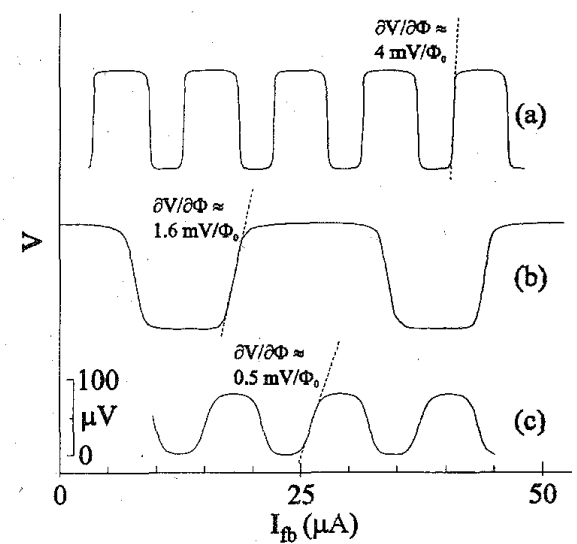

Fig. 4. Experimental $V-\Phi$ curves: (a) input coil open; (b) input coil shorted; (c) shielded first-order gradiometer connected. Traces (a) and (b) have been recorded at $I_{b} \approx 150 \mu \mathrm{A}$, and trace (c) at $I_{b} \approx 100 \mu \mathrm{A}$. See the text for more information.

inductance between the feedback coil and the signal SQUID, $M_{f b}$, differs for the three situations. With open input coil, $M_{f b, \text { open }}=220 \mathrm{pH}$, with shorted input coil, $M_{f b \text {, short }}=$ $80 \mathrm{pH}$ and with connected pick up coil, $M_{f b, p u}=183 \mathrm{pH}$. This is caused by screening of the signal SQUID inductance by the superconducting flux transformer. This effect has been used to estimate the inductances in the flux transformer circuit.

The amount of screening is reflected in the effective SQUID inductance, given by

$$
L_{s q, \mathrm{eff}}=L_{s q}-\frac{M_{\mathrm{in}}^{2}}{L_{\mathrm{in}}+L_{p u}+L_{\mathrm{par}}}
$$

where $L_{\text {par }}$ is the parasitic inductance in the flux transformer, mainly formed by the inductance of the twisted wires between the gradiometer and the input coil. Assuming that $M_{f b}=$ $k^{*} \cdot L_{s q \text {,eff }}$, the constant $k^{*}$ can be determined from the measurements with open flux transformer: $k^{*}=M_{f b \text {,open }} / L_{s q}=$ $220 \mathrm{pH} / 240 \mathrm{pH}=0.92$. In the case of a superconductively shorted input coil, $L_{s q \text {,eff, short }}=M_{f b \text {,short }} / k^{*}=87 \mathrm{pH}$. With shorted input coil, $L_{p u}+L_{\text {par }}=0$ in (9). Then, the

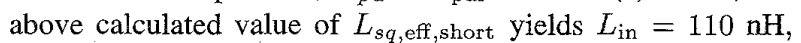
which is close to the design value of $100 \mathrm{nH}$. Analogously, $L_{p u}+L_{\text {par }}$ can be estimated from (9), using the results of the measurements with connected gradiometers, resulting in $L_{p u}+L_{\mathrm{par}}=0.31 \mu \mathrm{H}$. The fact that this is slightly smaller than the design value of $L_{p u}=0.35 \mu \mathrm{H}$ is mainly caused by screening of $L_{p i}$ by the $\mathrm{Pb}$ can, that fits very closely around the gradiometers.

A field-to-flux calibration has been performed after removal of the $\mathrm{Pb}$ shielding around the pick up coils. By positioning a known ac magnetic dipole at various distances from the gradiometers, the field-to-flux transfer was measured to be $\partial B / \partial \Phi=0.4 \mathrm{nT} / \Phi_{0}$. Here, $B$ is the difference between the magnetic field at the position of the sensing turns and the field at the position of the compensation turns of the gradiometer. This experimental transfer value corresponds to an effective

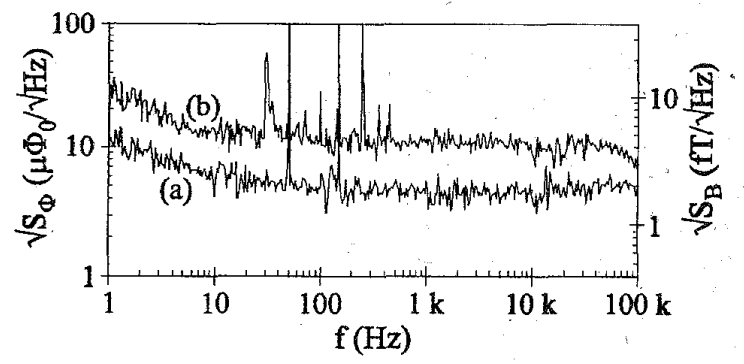

Fig. 5. FLL noise spectra: (a) open input coil; (b) first-order gradiometer connected. Both traces have been recorded at $I_{b} \approx 100 \mu \mathrm{A}$.

pickup area $A_{p u, \text { eff }}=5.2 \mathrm{~mm}^{2}$. Using

$$
\Phi_{s q}=B \cdot A_{p u} \cdot \frac{M_{\text {in }}}{L_{p u}+L_{\text {in }}+L_{\text {par }}}
$$

combined with $A_{p u}=6.3 \mathrm{~cm}^{2}, M_{\text {in }}=4.1 \mathrm{nH}$, and $L_{\text {in }}=$ $110 \mathrm{nH}$, it follows that $L_{p u}+L_{\mathrm{par}}=0.39 \mu \mathrm{H}$. This is a reasonable value in view of the design value of $L_{p u}=$ $0.35 \mu \mathrm{H}$ : it indicates a parasitic inductance of about $40 \mathrm{nH}$ for the $15 \mathrm{~cm}$ long twisted leads between gradiometer and DROS module.

\section{B. DROS Characterization}

The $V-\Phi$ characteristics in Fig. 4 show a large transfer; values of up to $4 \mathrm{mV} / \Phi_{0}$ have been observed at large bias currents (of the order of $150 \mu \mathrm{A}$, Fig. 4(a) and (b)). However, the experimental noise performance of the DROS's is optimum at lower bias currents, about $100 \mu \mathrm{A}$ typically, corresponding to a transfer of 0.5 to $1 \mathrm{mV} / \Phi_{0}$, as in Fig. 4(c). This is in good agreement with (2), which predicts an optimum bias current of $85 \mu \mathrm{A}$. For lower bias currents, the flux-to-voltage transfer decreases, so that amplifier noise limits the system sensitivity, while for larger bias currents, (2) is not satisfied anymore, causing the intrinsic DROS sensitivity to limit the system sensitivity, despite the high value of $\partial V / \partial \Phi$.

The positive effect of the external $R_{\text {in }}-C_{\text {in }}$ shunt across the input coil is reflected in a larger transfer and a better noise performance. The transfer improved by a factor of 3 and the white flux noise level decreased by a factor of 1.5 when the shunt was connected, probably because of damping of input coil resonances. Reduction of the value of $R_{\text {in }}$ resulted in an increasing white fiux noise level, due to the Johnson noise generated in $R_{\text {in }}$, despite the dc blocking capacitor $C_{\text {in }}$. Downmixing of high frequency noise is probably the mechanism involved.

A typical FLL flux noise spectrum of a DROS without pickup coil, with $R_{\mathrm{in}}-C_{\text {in }}$ shunt, is given in trace (a) of Fig. 5. The white noise level is $4.5 \mu \Phi_{0} / \sqrt{\mathrm{Hz}}$ typically, corresponding to $\varepsilon=275 \mathrm{~h}$. At $1 \mathrm{~Hz}$, the noise level is $10 \mu \Phi_{0} / \sqrt{\mathrm{Hz}}$. In accordance with (7), the bandwidth of the FLL is larger than $100 \mathrm{kHz}$, which is the maximum bandwidth of the spectrum analyzer (HP 3562A) that was used for the noise measurements.

The flux noise did not change significantly when the flux transformer was superconductively shorted, indicating that the 
noise characteristics of the DROS are not strongly influenced by the flux transformer circuit. However, when the shielded first-order gradiometers were connected, the flux-to-voltage transfer decreased and the white flux noise level rose to about $10 \mu \Phi_{0} / \sqrt{\mathrm{Hz}}$, as shown by the trace in Fig. 5(b). Probably, this is caused by RF interference, despite all precautions that were taken to minimize this source of disturbances. The right axis of Fig. 5 shows the magnetic field noise according to the experimental field-to-flux transfer of $0.4 \mathrm{nT} / \Phi_{0}$. As can be seen, the white noise level amounts about $4 \mathrm{fT} / \sqrt{\mathrm{Hz}}$, increasing to $10 \mathrm{fT} / \sqrt{\mathrm{Hz}}$ at $1 \mathrm{~Hz}$.

\section{Multichannel Operation}

A possible source of degradation of DROS characteristics in a multichannel system could be crosstalk at the relaxation frequency. During the relaxation oscillations, the current through the signal SQUID oscillates between zero and the critical current of the SQUID. This oscillating current causes the net flux in the SQUID to oscillate with an amplitude of the order of $1 \Phi_{0}$ with a frequency equal to the relaxation frequency, so about $1 \mathrm{GHz}$ in the present case. Via the flux transformer, these oscillations are transferred to the pickup section of the magnetometer, and finally, crosstalk between adjacent pick up coils can couple them to another channel, thus causing a high frequency ripple on the signal flux. Even with an $R_{\text {in }}-C_{\text {in }}$ shunt across the input coils of both DROS's, the amplitude of this ripple can be hundreds of $\mu \Phi_{0}$, so that interference of the two relaxation frequencies is not quite unrealistic.

In practice, when two adjacent DROS gradiometer channels were operated simultaneously, the spectral flux noise density of one channel was unaffected by the operation of the other DROS, which was biased in such a way that its signal SQUID was oscillating. Probably, the spectral impurity of the relaxation oscillation frequencies in both DROS's is large enough to "smear" the crosstalk flux over a considerable bandwidth, thus drowning it completely in the DROS noise of $4 \mathrm{fT} / \sqrt{\mathrm{Hz}}$. A low frequency crosstalk via the flux transformers was clearly present: when a feedback flux of $1 \Phi_{0}$ was applied to one DROS, the other one detected a flux of $0.005 \Phi_{0}$. This crosstalk of $0.5 \%$ is a direct consequence of the small distance between the gradiometers: they are spaced by less than $1 \mathrm{~mm}$.

These results show unambiguously that interference between the relaxation oscillation processes in two adjacent DROS channels does not deteriorate the DROS characteristics, despite crosstalk via the flux transformers. This opens the way to highly sensitive multichannel magnetometers based on DROS's.

\section{CONCLUSION}

The feasibility of a multichannel DROS magnetometer system has been investigated by constructing a three-channel DROS gradiometer setup with relatively simple roomtemperature FLL electronics. Typically, the bare DROS's that are used show a white flux noise level of $4.5 \mu \Phi_{0} / \sqrt{\mathrm{Hz}}$ in FLL. An external $R-C$ shunt across the input coil has a positive effect on both the flux-to-voltage transfer and the noise performance. After first-order wire-wound gradiometers had been connected, the flux noise level increased to $10 \mu \Phi_{0} / \sqrt{\mathrm{Hz}}$, probably due to high frequency electro-magnetic interference. With the experimental field-to-flux transfer of $0.4 \mathrm{nT} / \Phi_{0}$, this flux noise level corresponds to a white magnetic field noise of $4 \mathrm{fT} / \sqrt{\mathrm{Hz}}$. At $1 \mathrm{~Hz}$, the field noise amounts $10 \mathrm{fT} / \sqrt{\mathrm{Hz}}$. The noise level is not influenced by the relaxation oscillations in adjacent channels, thus showing that DROS's can be used in multichannel magnetometers.

\section{REFERENCES}

[1] D. Drung, "DC SQUID systems overview," Supercond. Sci. Technol., vol. 4, pp. 377-385, 1991

[2] C. D. Tesche and J. Clarke, "DC SQUID: Noise and optimization," Low Temp. Phys., vol. 29, pp. 301-331, 1977.

[3] D. Drung, R. Cantor, M. Peters, H. J. Scheer, and H. Koch, "Low-noise high speed dc superconducting quantum interference device magnetometer with simplified feedback electronics," Appl. Phys. Lett., vol. 57, pp. $406-408,1990$.

[4] D. Drung and H. Koch, "An integrated dc SQUID magnetometer with variable additional positive feedback," Supercond. Sci. Technol., vol. 7, pp. $242-245,1994$.

[5] M. Gershenson, "Design of a hysteretic SQUID as the readout for a dc SQUID," IEEE Trans. Mag., vol. 27, pp. 2910-2912, 1991.

[6] V. Foglietti, "Double dc SQUID for flux-locked-loop operation," Appl. Phys. Lett., vol. 59, pp. 476-478, 1991

[7] R. P. Welty and J. M. Martinis, "Two-stage integrated SQUID amplifier with series array output," IEEE Trans. Appl. Superconduct., vol. 3, pp. 2605-2608, 1993.

[8] N. Fujimaki, H. Tamura, T. Imamura, and S. Hasuo, "A single-chip SQUID magnetometer," IEEE Trans. Electron Devices, vol. 35, pp. 2412-2418, 1988.

[9] M. Mück and C. Heiden, "Simple dc-SQUID system based on a frequency modulated oscillator," IEEE Trans. Magn., vol. 25, pp. 1151-1153, 1989.

[10] M. Mück, H. Rogalla, and C. Heiden, "A frequency-modulated read-out system for dc SQUIDs," Appl. Phys., vol. A 47, pp. 285-289, 1988.

[11] J. Kawai, G. Uehara, M. Mizutani, Y. Kondo, N. Harada, and H. Kado, "Relaxation oscillating SQUIDs using $\mathrm{Nb} / \mathrm{AlOx} / \mathrm{Nb}$ Josephson tunnel junctions," in Superconducting Devices and Their Applications, H. Koch and H. Lübbig, Eds. Berlin: Springer-Verlag, 1991, pp. 341-344.

[12] D. J. Adelerhof, H. Nijstad, J. Flokstra, and H. Rogalla, "(Double) relaxation oscillation SQUIDs with high flux-to-voltage transfer: Simulations and experiments," J. Appl. Phys., vol. 76, pp. 3875-3886, 1994.

[13] D. J. Adelerhof, M. J. van Duuren. J. Flokstra, and H. Rogalla "Second generation de SQUID sensors: ROS with frequency readout and DROS with voltage readout," in SPIE Proc., vol. 2160, pp. 142-153, 1994.

[14] D. J. Adelerhof, H. Nijstad, J. Flokstra, and H. Rogalla, "Relaxation oscillation SQUIDs with high $\mathrm{dV} / \mathrm{d} \Phi, "$ IEEE Trans. Appl. Superconduct. vol. 3, pp. 1862-1865, 1993.

[15] D. J. Adelerhof, J. Kawai, G. Uehara, and H. Kado, "High sensitivity double relaxation oscillation superconducting quantum interference devices with large transfer from flux to voltage," Rev. Sci. Instrum., vol. 66, pp. 2631-2637, 1995.

[16] , "High sensitivity double relaxation oscillation superconducting quantum interference devices," Appl. Phys. Lett, vol. 65, pp. 2606-2608, 1994.

[17] D. J. Adelerhof, J. Kawai, K. Tsukada, G. Uehara, and H. Kado, "Magnetometers based on double relaxation oscillation superconducting quantum interference devices," Appl. Phys. Lett. vol. 66, pp. 2274-2276, 1995.

[18] J. M. Jaycox and M. B. Ketchen, "Planar coupling scheme for ultra low noise de SQUIDs," IEEE Trans. Magn., vol. MAG-17, pp. 400-403, 1981:

[19] K. Enpuku, K. Sueoka, K. Yoshida, and F. Irie, "Effect of damping resistance on voltage versus flux relation of a dc SQUID with large inductance and critical current," J. Appl. Phys., vol. 57, pp. 1691-1697, 1985.

[20] W. Jaszczuk, H. J. M. ter Brake, J. Flokstra, D. Veldhuis, R. Stammis, and H. Rogalla, "Bonding of a niobium wire to a niobium thin film," Meas. Sci. Technol., vol. 2, pp. 1121-1122, 1991. 
Michiel J. van Duuren received the M.Sc. degree in applied physics from the University of Twente, The Netherlands, in 1993. The subject of his graduation report was frequency readout of relaxation oscillation SQUID's.

Since graduation, he has continued the (D)ROS research as a Ph.D. student at the University of Twente.

Y. H. Lee, photograph and biography not available at the time of publication.

Derk Jan Adelerhof received the Ph.D. degree from the University of Twente, The Netherlands, in 1993. His dissertation was on double relaxation oscillation SQUID's.

He entered the field of superconductivity in 1988 when he studied the growth of thin-film $\mathrm{YBaCuO}$ layers by laser ablation. He continued the study of (D)ROS's for his postdoctoral work, partly at the University of Twente, The Netherlands, and partly at the Supcrconducting Sensor Laboratory, Japan. In that period he was also involved in the X-ray detector project at the University of Twente. At present, he is with the Philips Research Laboratory in The Netherlands.

J. Kawai, photograph and biography not available at the time of publication.
H. Kado, photograph and biography not available at the time of publication.

Jaap Flokstra received the M.Sc. degree in applied physics from the Technical University of Delft, The Netherlands, in 1971, and the. Ph.D. degree in applied physics from the University of Twente, The Netherlands, in 1977. He has been an Associate Professor in the Department of Applied Physics, University of Twente, since 1985 . His current research interest is in the applications of low and high Tc superconductivity such as X-ray detectors, dc SQUID's, gravity gradiometers, etc. He has been the Dean of Research of the faculty of Applied Physics at Twente since 1993.

Horst Rogalla received the Ph.D. degree in 1979 in Munster, Germany.

He joined the faculty of Physics in Giessen, Germany, in 1977. Since 1987 he has been a Professor at the Department of Applied Physics, University of Twente, The Netherlands, and is the Head of the Low Temperature Division His research interests are in superconducting electronics and material science, especially related to thin film growth and properties.

Dr. Rogalla is a member of the Dutch, German, and American Physical Society as well as the American and European Materials Research Society. 